\section{Photonic Transistor Realized with Local Plasmon Amplification}

Researchers from the Laboratory for Advanced Optical Technology at the National Institute of Advanced Industrial Science and Technology (AIST), the Advanced Technology Research Laboratory at Sharp Corporation, and the Data Storage Technology Center at TDK Corporation have demonstrated a system that has the potential to realize all-thin-film photonic transistors by using local plasmon amplification. Using a technique called super-resolution near-field structure (super-RENS), which was originally developed for optical near-field recording, surface plasmons can be generated, without a prism, by using a specially designed multilayer with a focused laser beam.

In the April 23 issue of Applied Physics Letters, J. Tominaga of AIST and co-workers describe a procedure for fabricating a plasmon transistor as well as the experimental setup used to confirm the device. By focusing both a red (635-nm) and a blue (405-nm) laser beam into one small spot on a high-speed rotating optical disk, a large signal enhancement was observed. They found that a plasmon interaction generated between silver light-scattering center and small marks in the optical disk, recorded with a superRENS, produced a large signal amplification in the spot $(<1 \mathrm{~mm})$. The silver lightscattering center was formed by the local decomposition of a silver oxide layer. A modulated signal of the blue laser was enhanced $60 \times$ by controlling the red-laser power from $1.5 \mathrm{~mW}$ to $3.5 \mathrm{~mW}$. By tuning the size of the light-scattering center, through control of the laser power responsible for generating the scattering center, the signal released from the plasmon reservoir was controlled. In their system, the light-scattering center plays the role of a gate in the photonic transistor.

The researchers' results indicate that a photonic transistor is possible by using local plasmon scattering. The local plasmon photonic transistor has advantages over photonic devices that use surface plasmons. For example, prisms, which were previously required to attain attenuated total reflection, are replaced by a thin film of silver oxide. This enables the devices to be manufactured thinner and smaller, and at a reduced cost, through a combination of micromachining and thinfilm technologies. Tominaga believes that "all-thin-film photonic plasmon circuits are no longer a dream, but rather a technology which may soon be manufactured like conventional electronic devices."

STEFFEN K. KALDOR

\section{Optical Recording Fabricated with Dye-} Doped Polymer-Dispersed LC Films

A group of researchers from the National Cheng Kung University and the Fortune Institute of Technology in Taiwan has devised a method of recording optical holograph permanent gratings that are electrically switchable. As reported in the April 1 issue of Optics Letters, this technique has a combination of high light sensitivity and short recording time.

The recording medium is a homogeneous mixture of liquid crystals, a prepolymer (called NOA65), and a dye (methyl red). Two laser beams ( $\lambda=532 \mathrm{~nm}, \sim 6 \mathrm{~ns}$ pulses) from a single $Q$-switched Nd:YAG source write the holographic grating. The polymer is then UV-cured at $\sim 11.5 \mathrm{~mW} / \mathrm{cm}^{2}$.

The dye molecules exert a torque on the liquid crystals where they are photoexcited by the laser. This action causes the alignment of the liquid crystals along their director axis. Liquid crystals remain in random orientation in the "unwritten" regions, as confirmed by both polarization and diffraction experiments. According to the researchers, the ease of sample preparation, the good light sensitivity, and the fast recording time of this technique may lead to many future advancements in holography. Although the diffraction grating was "permanent," diffraction could be turned off by applying an ac rms voltage of $140 \mathrm{~V}$ at $1 \mathrm{~Hz}$.

JUNE LAU

\section{Seeding of the Reaction-Bonded Aluminum Oxide Process with Submicron $\mathrm{Al}_{2} \mathrm{O}_{3}$ Particles Reduces the Transformation Temperature}

A team of researchers at The Pennsylvania State University has demonstrated that in the production of ceramics, seeding the reaction-bonded aluminum oxide (RBAO) process with $\sim 0.2-\mu \mathrm{m}$-sized $\alpha-\mathrm{Al}_{2} \mathrm{O}_{3}$ particles decreased the phasetransformation temperature to $\sim 962^{\circ} \mathrm{C}$. The fine microstructure of the compacts decreases the sintering temperature and improves the densification.

"Reaction-based processing is a novel approach for the fabrication of ceramics and ceramic-matrix composites," said Gary Messing, professor of materials science and engineering at Penn State. "The process is very attractive, as it is environmentally benign and gives compacts with a high green strength and a low net shrinkage."

In the RBAO process, which was developed in 1989, a mixture of aluminum and $\alpha-\mathrm{Al}_{2} \mathrm{O}_{3}$ powders is used to produce dense $\alpha-\mathrm{Al}_{2} \mathrm{O}_{3}$-based composites. After attrition milling, the resulting slurry is dried and pressed into pellets. Upon heating, the $\mathrm{Al}$ is oxidized and forms $\gamma-\mathrm{Al}_{2} \mathrm{O}_{3}$. Further heating leads to transformation to $\alpha-\mathrm{Al}_{2} \mathrm{O}_{3}$ above $1100^{\circ} \mathrm{C}$ and sintering above $1550^{\circ} \mathrm{C}$.

"There was some evidence in our earlier work that the $\gamma-\mathrm{Al}_{2} \mathrm{O}_{3}$ to $\alpha-\mathrm{Al}_{2} \mathrm{O}_{3}$ transformation can have a significant effect on sintering kinetics, temperature, and microstructure," said Ender Suvaci, who did his PhD research on RBAO with Messing before accepting a position as an assistant professor at Anadolu University in Turkey. " $\gamma-\mathrm{Al}_{2} \mathrm{O}_{3}$ can be seeded with $\alpha-\mathrm{Al}_{2} \mathrm{O}_{3}$ to decrease the sintering temperature, so we decided to investigate whether the RBAO process could be seeded by using fine $\alpha-\mathrm{Al}_{2} \mathrm{O}_{3}$ starting particles, too."

As reported in the March issue of the Journal of the American Ceramic Society, the team carried out comparative studies on two composites using coarse ( 6-10- $\mu \mathrm{m})$ and fine $(\sim 0.2-\mu \mathrm{m}) \alpha-\mathrm{Al}_{2} \mathrm{O}_{3}$ starting powders. This resulted in a higher seed frequency than the intrinsic nucleation density $\left(2.9 \times 10^{14}\right.$ as compared to $1.1 \times 10^{11}$ seeds $/ \mathrm{cm}^{3} \gamma-\mathrm{Al}_{2} \mathrm{O}_{3}$ ) for the fine powder, while the value for the coarse powder is lower $\left(1.1 \times 10^{10}\right.$ seeds $\left./ \mathrm{cm}^{3} \gamma-\mathrm{Al}_{2} \mathrm{O}_{3}\right)$. The transformation of the as-formed $\gamma-\mathrm{Al}_{2} \mathrm{O}_{3}$ to $\alpha-\mathrm{Al}_{2} \mathrm{O}_{3}$ started at $963^{\circ} \mathrm{C}$ and $1052^{\circ} \mathrm{C}$ for the fine and coarse mixtures, respectively, demonstrating the successful seed effect. Sintering was observed at temperatures as low as $1135^{\circ} \mathrm{C}$ in the composite using fine $\alpha-\mathrm{Al}_{2} \mathrm{O}_{3}\left(\sim 127^{\circ} \mathrm{C}\right.$ lower than in the coarse sample). The smaller particle size resulted in better densification during sintering.

Messing said, "Although shown for $\mathrm{RBAO}$, it is clear that seeding is effective for other reaction-based ceramics and especially mixed metal-oxide ceramics where the phase transformation is nucleation-controlled."

CORA LIND

\section{Real-Time X-Ray Microbeam \\ Technique Used to Study Electromigration in $\mathrm{Al}(\mathrm{Cu})$ \\ Conductor Lines}

Researchers from the IBM T.J. Watson Research Center in Yorktown Heights, NY, and the Microelectronics Division in East Fishkill, NY, have developed an x-ray microtopography technique for studying stresses and interface integrity in thinfilm/semiconductor substrate composites. Using this technique, they have measured the evolution of electromigration-induced stress gradients and $\mathrm{Cu}$ concentration in $\mathrm{Al}(\mathrm{Cu})$ conductor lines. This in situ method, which has a strain sensitivity of $10^{-7}$, allows for detection of the stress gradient at both low current densities $\left(10^{4}-10^{5} \mathrm{~A} / \mathrm{cm}^{2}\right)$ and early times.

As reported in the April 30 issue of Applied Physics Letters, thin-film $\mathrm{Al}-0.25 \% \mathrm{Cu}$ conductor lines $(200 \mu \mathrm{m}$ long $\times 10 \mu \mathrm{m}$ wide $\times 0.5 \mu \mathrm{m}$ thick) deposited on silicon (100) substrates and passivated 
with a $1.5 \mu \mathrm{m} \mathrm{SiO}$ layer were studied with both $x$-ray microtopography and energydispersive fluorescence analysis. A 5- $\mu \mathrm{m}$ diameter $x$-ray spot was obtained by using a tapered glass capillary to focus $x$-rays from a beamline of the National Synchrotron Light Source at Brookhaven National Laboratory. The combination of the 5- $\mu \mathrm{m}$ diameter x-ray spot and a sample translation capability of $\pm 1 \mu \mathrm{m}$ allowed for spatially resolved measurements along the conductor line length. P.-C. Wang and coworkers said that unlike traditional x-ray stress measurements that rely on the change in a material's lattice parameter for strain information, this topography technique is based on mapping a suitable substrate reflection [in this case, $\mathrm{Si}(004)$ ] over the region covered by the thin film. The shear-stress maxima present at the film-substrate interface increase the bandpass of the substrate crystal and give rise to enhanced diffracted intensities. The observed contrast between diffracted intensities over different regions of the specimen can be correlated with the stress field in the film. A scintillation and a solidstate $\mathrm{Si}(\mathrm{Li})$ detector were used simultaneously to collect the Si diffraction and $\mathrm{Cu} \mathrm{K}{ }_{\alpha}$ fluorescence signals, respectively.
At $302^{\circ} \mathrm{C}$, an electrical current $\left(1 \times 10^{5}\right.$ $\mathrm{A} / \mathrm{cm}^{2}$ ) was passed through the conductor line, and the sample was scanned repetitively while topography and fluorescence data were collected. The researchers' results illustrate changes in the stress field and $\mathrm{Cu}$ concentration for both the early and late stages of electromigration, as well as the post-relaxation (current turned off) stage. During the initial $5 \mathrm{~h}$ of electromigration, $\mathrm{Cu}$ was observed to migrate along the direction of electron flow, eventually forming a continuous monotonic concentration gradient. The diffracted Si intensity was enhanced both at the cathode, due to an increase in tensile stress, and at the anode, due to the local compressive stress from the mass pileup there. Measurements in the steady-state regime $(\sim 19 \mathrm{~h})$ provided additional information about the local stress profile and the formation of lateral extrusions near the anode region (see figure). After switching off the current, it was observed that $\mathrm{Cu}$ flowed back into the depleted regions, resulting in a more uniform $\mathrm{Cu}$ distribution, and the electromigration-induced stress gradient was significantly reduced along the line, except at locations where permanent damage had occurred.

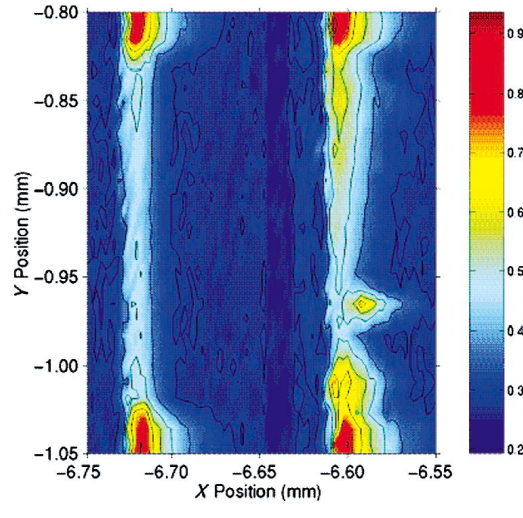

Figure. Intensity contours of Si(004) diffraction over an area containing two $\mathrm{Al}(\mathrm{Cu})$ wires, with the electromigrated wire on the right and the control sample on the left. The $\mathrm{Cu} \mathrm{K}_{\alpha}$ plot is in a log scale, and the Si(004) plot is in a linear scale, with both intensities normalized by the monitor intensity upstream. Reproduced with permission from 2001 Applied Physics Letters, 78(18), 2712.

"We are very excited about being able to measure the real-time evolution of electromigration stress gradients and $\mathrm{Cu}$ migration simultaneously, especially at such low

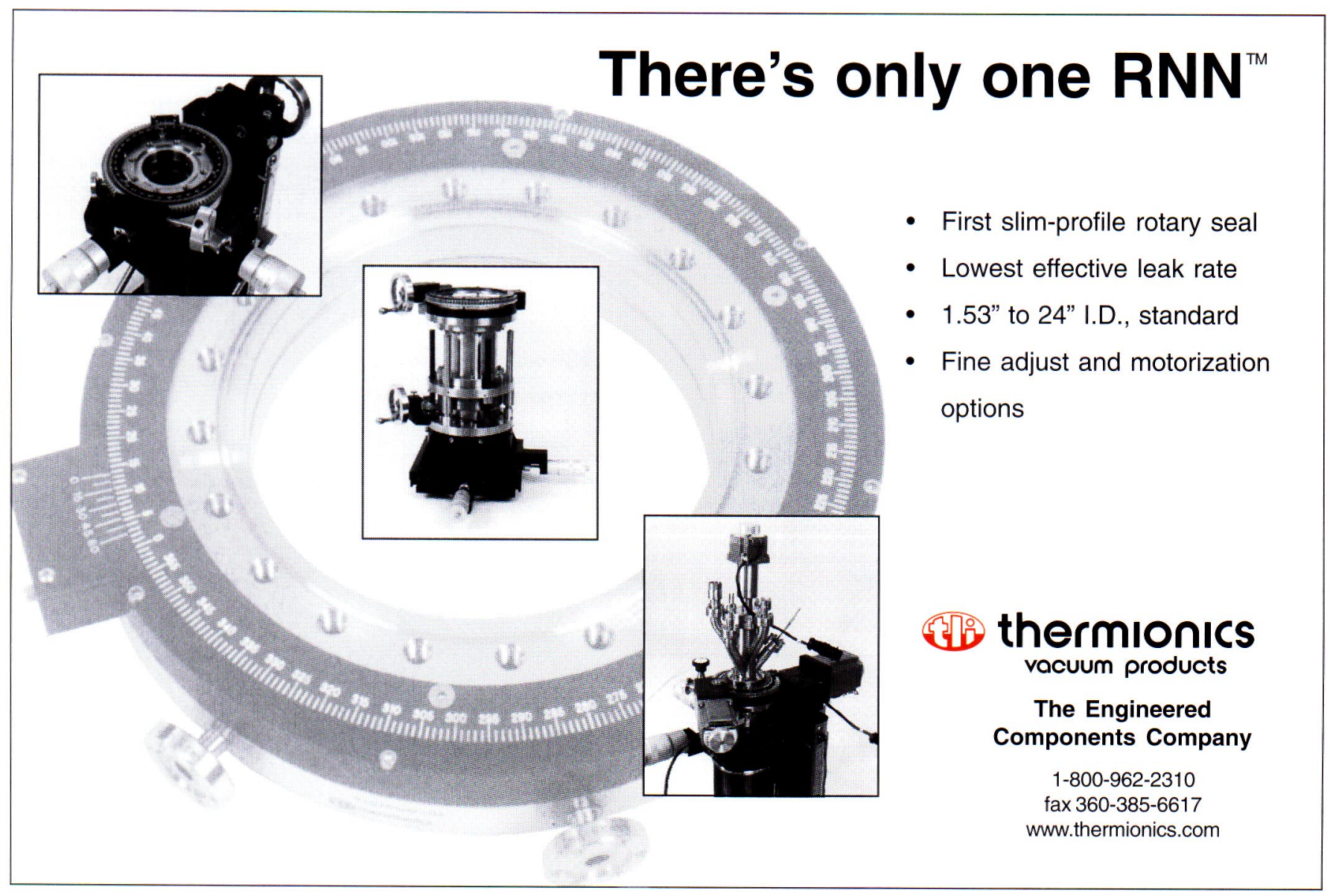

Circle No. 5 on Inside Back Cover 
current densities and early times," said the researchers. "We are hopeful that this technique will help us to better understand some of the fundamental issues associated with electromigration."

\section{Silver in Hybrid Membranes Facilitates Separation of Ethene and Ethane}

Researchers at the Osaka National Research Institute in Japan have achieved good selectivity of ethene against ethane in separation processes using inorganicorganic hybrid membranes containing sil$\operatorname{ver}(\mathrm{I})$ ions. The organic poly $(\mathrm{N}$-vinylpyrrolidone) (PVP) part increased the flexibility of the inorganic network and immobilized the silver ions, which can function as olefin carriers. The membranes showed higher selectivity at higher temperatures, as thermal energy enhances the decomplexation rate of the silver olefin complexes.

Separation of paraffins and olefins is usually carried out by cryogenic distillation. Among the alternatives that were investigated to replace this energy-intensive process, the use of facilitated support membranes containing $\mathrm{Ag}^{+}$ions seemed to be promising. While supported liquid membranes and ion-exchange membranes require saturation of the feed gas with solvent, silver polymer membranes can be used to separate paraffins and

\section{Cost-Effective Portable Spin Goaters}

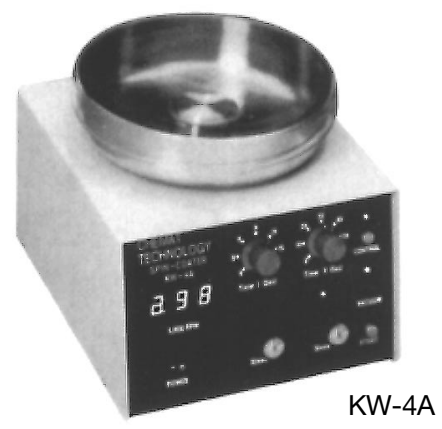

\section{Two-Stage Spinning \\ - Dispense liquid during Stage 1 \\ - Spin-up and flatten during Stage 2}

\section{Adjustable Speed}

Stage 1

- 500 to $2,500 \mathrm{rpm}$

- 2 to 18 seconds

Stage 2

- 1,000 to $8,000 \mathrm{rpm}$

- 3 to 60 seconds

\section{Precision Video Biologital Microseope}

Only \$1,968.90

(including Video Camera)

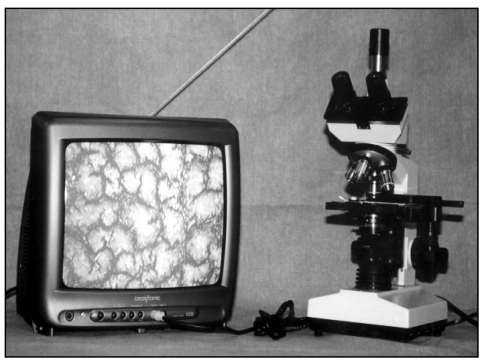

XSZ-107CCD

\section{Features}

- Fully Coated Optical System

- 45mm Achromatic Objective, Parfocall

- Coaxial Coarse and Fine Focus Adjustment

- Focusing Stops to prevent Objectives \& Slides from being

Damaged

- Built-in Illumination, Adjustable

- Brightness

Specifications

- Trinocular Head for Video Camera

- Wide Field Eyepieces WF 10X, P16X( WF 16X)

- Achromatic 4X, 10X, 40X(S) and 100X(S, Oil)

Fax: 818-727-9477 • E-mail: chemat@aol.com

www.chemat.com

olefins without water. The limitation of these membranes to low temperatures has now been overcome by replacing the pure polymer membrane with an organic-inorganic hybrid membrane as reported in the March issue of the Journal of the American Ceramic Society.

The hybrid membranes were prepared via a sol-gel route using tetraethoxysilane, propyl triethoxysilane, water, and $\mathrm{HNO}_{3}$. After several hours of stirring, PVP and later $\mathrm{AgBF}_{4}$ were added. Membranes were produced by dip-coating on a porous aluminum tube. Two membranes containing $\sim 10 \mathrm{wt} \%$ PVP, one with and one without silver, as well as a membrane containing $\sim 20 \mathrm{wt} \%$ PVP and silver ions, were prepared. Fourier transform infrared spectrophotometry measurements suggested that the silver ions were bound to the amide group of the PVP segments. The silica matrix gave the membranes enhanced thermal stability; the PVP made the inorganic network more flexible and nonporous. While the membrane without silver ions showed little selectivity for ethene, both of the silver-containing membranes gave $P_{\mathrm{C}_{2} \mathrm{H}_{4}} / P_{\mathrm{C}_{2} \mathrm{H}_{6}}$ ratios that increased with increasing temperature. At $423 \mathrm{~K}$, values of 1.9 and 2.3 were observed for the membranes containing $10 \mathrm{wt} \%$ and $20 \mathrm{wt} \%$ PVP, respectively.

The researchers said that selectivity of the membranes could be improved by, "(1) raising the temperature to increase the decomplexation rate of $\mathrm{C}_{2} \mathrm{H}_{4}$ from $\mathrm{Ag}^{+}$ and (2) increasing the PVP content." The researchers attribute the latter effect to the ability of the PVP to increase the flexibility of the inorganic framework and the immobilization of $\mathrm{Ag}^{+}$in the polymer segments. CORA LIND

\section{Mesoscopic Hollow Titania \\ Spheres Functionalized on Inner Surface with Silver Nanocrystals}

Mesoscopic hollow spheres have potential applications in the fields of catalysis, drug delivery, and coatings. The preparation of hollow titania mesospheres with interior surfaces functionalized with silver nanocrystals was recently reported by Younan Xia and co-workers from the Departments of Chemistry and Materials Science and Engineering at the University of Washington-Seattle. The materials were formed by decorating the surfaces of polystyrene (PS) spheres with nanocrystals and then using the modified spheres as templates for the sol-gel synthesis of titania (see figure). This method allows for facile synthesis of large quantities of hollow spheres with precisely controlled void size, shell thickness, and inner surface functionalization.

As reported in the April issue of Chemistry of Materials, commercially pur- 